Dynamic order acceptance and capacity planning within a multi-project environment

Jade Herbots, Willy Herroelen and Roel Leus

DEPARTMENT OF DECISION SCIENCES AND INFORMATION MANAGEMENT (KBI) 


\title{
Dynamic Order Acceptance and Capacity Planning within a Multi-Project Environment
}

\author{
Jade Herbots • Willy Herroelen • Roel Leus \\ Department of Decision Sciences and Information Management, \\ Katholieke Universiteit Leuven, Belgium \\ \{Jade.Herbots ; Willy.Herroelen ; Roel.Leus\}@econ.kuleuven.be
}

We present a tactical decision model for order acceptance and capacity planning that maximizes the expected profits from accepted orders, allowing for regular as well as non-regular capacity. We apply stochastic dynamic programming to determine a profit threshold for the accept/reject decision as well as an optimal capacity allocation for accepted projects, both with an eye on maximizing the expected revenues within the problem horizon. We derive a number of managerial insights based on an analysis of the influence of project and environmental characteristics on optimal project selection and capacity usage.

Keywords: order acceptance, capacity planning, multi-project, stochastic dynamic programming.

\section{Introduction}

A lot of companies tend to accept all projects with a positive net present value (NPV), without consideration of the effect on the planning of the already accepted projects. In case of external projects (projects performed for customers external to the organization), this is often the consequence of the functional separation between the order-acceptance decision, which is made by the sales department, and capacity planning, which usually lies in the hands of the production department. These two departments generally have conflicting objectives: in order to boost sales, one should try to accept as many projects as possible, while production attempts to live up to promised delivery dates. This divergence of interests can result in considerable delays, violated due dates and/or excessive use of highly expensive non-regular capacity such as overtime and temporary labor. It is therefore essential that project selection and planning be integrated (Zijm 2000).

This paper examines the simultaneous dynamic order-acceptance and capacity-planning decision. Order acceptance refers to the accept/reject decision an over-demanded company has to make upon project arrival. Capacity planning is concerned with making a rough 
sketch of the resource usage (regular and non-regular) and the timing of the work packages of both accepted as well as candidate projects.

In a multi-project environment, projects typically share common resources. Adequate management of these scarce resources is therefore of crucial importance. Consequently, the development of good acceptance rules and capacity-planning tools is extremely relevant, as they can support decisions such as due-date quotation, price quotation and hiring non-regular capacity. Appropriate order acceptance and capacity planning allows to gain a larger control over the use of non-regular capacity, increase profits and improve delivery performance, which creates a competitive advantage to the company. These benefits constitute the motivation for this research.

Most of the existing literature on order acceptance and capacity planning deals with static models, in which project selection is performed only once, at the beginning of the planning horizon. Although some models also consider the possibility of intermediate action, they are mainly suitable for internal project selection, where the set of projects available for execution during the planning horizon is known in advance. By internal projects, we refer to projects that have been proposed by internal customers, examples are internal R\&D (Research and Development) or NPD (New Product Development) projects. As a consequence, the static approach is less realistic when dealing with external projects which, in general, arise dynamically to the organization and require immediate response; models specific to this situation are called dynamic models. This paper is concerned with the development of a dynamic model for dealing with external project arrivals.

The issue of project selection can be positioned at the tactical or strategic decisionmaking level and is part of project portfolio management, which is concerned with project selection and prioritization by executive and senior management, with a focus on strategic medium- and long-term decisions. If the financial implications of individual projects have a considerable impact on the vitality of the organization, project selection is of strategic importance. Additionally, strategic decision making is also concerned with setting global capacity levels, the location of new sites, etc. At the tactical level, we encounter problems such as selecting non-strategic projects, capacity planning, due-date setting, order bidding, etc.

In this paper, we present a tactical decision model for order acceptance and capacity planning that maximizes the expected profits from accepted orders under limited regular per-period capacity; additionally, non-regular capacity units can be allocated at specific 
per-unit costs. At the completion of a project, the company receives a payoff which it can reinvest until the end of the problem horizon at a specified interest rate. We assume that the company has forecasts for the main features of the incoming projects. Our models are particularly relevant for MTO (manufacture-to-order) and construction environments, where at least rudimentary information about the work content of future projects is available.

The contributions of this text are the following. First, we introduce a formal problem statement. Next, we apply stochastic dynamic programming (SDP) to determine a profit threshold for the accept/reject decision, as well as an optimal capacity allocation for accepted projects, both with an eye on maximizing the expected revenues within the problem horizon. Finally, using the SDP formulation, we derive a number of managerial insights based on an analysis of the influence of project and environmental characteristics on optimal project selection and capacity usage. For example, we investigate the effect of reinvestment revenues and of rush orders and we quantify the value of non-regular capacity units.

The remainder of this paper is organized as follows. The next section contains an overview of the literature on project selection, in which we discuss both static and dynamic models. In the third section, we introduce the basic problem characteristics and give an extended problem description. Section 4 contains a presentation of our SDP models. In Section 5 , we use the developed models to derive a number of important insights, and we distinguish the influence of the different problem characteristics. We determine the circumstances under which 'short-cut' planning rules exist and quantify the value of capacity and due date flexibility. In the sixth section we assess the computational performance of the model and finally, in Section 7, we draw some conclusions and look at future research opportunities.

\section{Literature survey}

Project selection has been studied in a broad variety of research domains, among which operations management, finance and managerial economics. In this section we discuss the relevant literature for the static and dynamic selection problem primarily in operations management but also in the other two cited literature streams. Several exact and approximate selection and planning methods have been proposed for the static problem; this literature is the topic of the next paragraph. As for the dynamic context, the existing work is relatively scarce and will be discussed in the second paragraph. 


\subsection{The static selection problem}

Static project selection implies the optimization of qualitative factors (e.g., alignment with company strategy), quantitative criteria (e.g., return on investment, NPV) or a combination of both. Especially for strategic decision making, the objectives will often include qualitative factors in addition to merely quantitative profitability measures. A large part of the literature is dedicated to $\mathrm{R} \& \mathrm{D}$ environments. In $\mathrm{R} \& \mathrm{D}$, the encountered decision problems are mostly strategic, given that effective $R \& D$ portfolio management is a prerequisite for the medium and long-term success of technology-driven organizations (Cooper et al. 1999). Surveys of R\&D project selection are presented by Baker \& Freeland (1975), Hall \& Nauda (1990) and Henriksen \& Traynor (1999). Henriksen \& Traynor (1999) categorize a wide range of tools with varying metrics and selection methods. In the following paragraphs, we provide a brief overview of the most-employed methods, namely mathematical programming, scoring and sorting methods, financial models and mapping.

Early attempts to tackle static selection usually took the form of mathematical-programming models related to knapsack formulations, for an overview we refer to Weber et al. (1990). Static selection is regarded here as the evaluation of a set of candidate projects, where the goal is to select a subset of projects that maximizes some objective function without violating the constraints. Integer-programming formulations are used by Beaujon et al. (2001), Golabi (1987) and Bard et al. (1988). Some more extended models take payoff interactions between projects into account (interdependencies between the financial benefits; Fox et al. 1984, Dickinson et al. 2001) as well as technical interactions (overlap between project contents; Czajkowski \& Jones 1986).

Baker \& Freeland (1975) assess why few quantitative models for R\&D project selection and capacity allocation have been implemented by managers. As a first reason, they mention the incapability of the models to capture all important aspects of the R\&D environment. A second problem is the quantification of the qualitative features of projects. Both drawbacks urge managers for scepticism about the outcome of the models. Hall \& Nauda (1990) found that the data required by the models is unavailable in most cases. The observation that mathematical-programming tools have not found widespread acceptance in practice has been confirmed more recently by Loch et al. (2001).

Other ways of approaching the static selection problem are scoring and sorting models. These models evaluate projects based on financial or non-financial measures. Projects are 
ranked via a score determined by e.g. analytical hierarchy process (AHP) (see Saaty 1994, Brenner 1994). Other scoring methods were developed by Henriksen \& Traynor (1999) and Eilat et al. (2006).

Financial models for portfolio selection often start from the Markowitz model, which minimizes the variability of the return of a portfolio subject to bounds on the expected return (see e.g. Luenberger 1998). Application of this model to R\&D portfolio selection has been suggested in the literature (Fox et al. 1984, Weber et al. 1990, Ringuest et al. 2004). In his dissertation, Jørgensen (1999) gives an extensive overview of the literature on financial methods applied to project selection.

Yet another angle to approach the portfolio selection problem can be found in mapping techniques. These are graphical and charting techniques that evaluate qualitative measures by visualizing the balance of the portfolio. Most of this literature descends from the disciplines of strategy and marketing. Wheelwright \& Clark (1992) give practical advice on how to organize the product development process.

The foregoing techniques mainly applied to strategic decisions. At a lower decision level we encounter the so-called Rough-Cut Capacity Planning (RCCP) problem, which is a specific type of tactical capacity planning. With RCCP, work packages can be executed at a variable intensity (De Boer 1998, Hans 2001, Kis 2005). These mathematical-programming models can be employed during the negotiation phase preceding the acceptance decision, but are usually invoked after project selection.

Detailed operational scheduling, relating to even shorter time horizons and higher planning frequencies than tactical models, is performed at the operational decision level (Demeulemeester \& Herroelen 2002). An example of operational project selection can be found in Yang \& Sum (1997). Within the operational domain of job-shop planning, job selection has been a topic of growing interest in the last decade. We refer to De et al. (1993) and Slotnick \& Morton (1996), who consider a pool of orders and separate sequencing and order acceptance. Lewis \& Slotnick (2002) extend the models to multiple periods. In our opinion, the lack of information one is usually confronted with when a project is initially presented to a company, makes such methods unfeasible for practical multi-project planning.

\subsection{The dynamic selection problem}

Dynamic project selection has been studied to a lesser extent than its static counterpart. Nevertheless, a broad variety of solution methods has been proposed. 
A basic approach to dynamic selection can be found in the dynamic stochastic knapsack developed in Kleywegt et al. (1998, 2001). This problem is an extension of Ross \& Tsang's (1989) stochastic knapsack problem to the case where items arrive over time with an unknown size and reward.

Queueing approaches to multi-project planning were introduced by Adler et al. (1995) and Levy \& Globerson (1997). The NPD process is modelled as a stochastic processing network in which engineering resources are 'workstations' and projects are 'jobs' that flow between the workstations. Both sources analyze the crucial issues responsible for time delays and cost overruns. De Reyck (1998) points out that the resulting analysis will not produce any detailed scheduling information on when to initiate or terminate individual activities or entire projects, but only allows for estimation of the average time spent on a single project. Kavadias \& Loch (2004) and Lewis et al. (1999) treat the dynamic selection problem as an admission control problem, a known problem within queueing theory.

Recently, Ebben et al. (2005) used simulation to compare different order-acceptance strategies in a job-shop environment. A similar method was proposed by Wester et al. (1992) and Akkan (1997) for production-to-order environments; in addition, they developed heuristics for scheduling the accepted work orders. In a completely different context, Balakrishnan et al. (1996) implement a decision-theory-based approach that reserves parts of the capacity for specified order types through a capacity allocation policy.

Most similar to the models described in this text is the work of Perry \& Hartman (2004), who examined the problem of selecting a number of orders with fixed production schemes from a set of arriving project offers. They solve a specific multi-knapsack problem through SDP. The suggested method determines the order combination that maximizes the expected future profits. Their model, however, does not define exact acceptance criteria nor does it allow the planner to deviate from the fixed production schemes. Moreover, the allocation of non-regular capacity units is not considered.

\section{Problem description}

In this section we present a detailed problem statement and explain how our assumptions translate to a practical multi-project setting. We introduce the major characteristics of an incoming project, namely its revenue, deadline and workload, we describe our assumptions regarding the order-arrival process, and we elaborate how capacity utilization is modelled. 
A comprehensive illustration is provided in the final paragraph.

\subsection{Project characteristics}

Project acceptance and capacity planning is confronted with restricted resource availability. We express both the project workload as well as the capacity available in the organization in discrete capacity units (e.g. man-hours). We refer to a single capacity unit belonging to the work content of a project as a work package.

It is standard practice for project management to structure the work content into small manageable elements as a result of the development of a work break down structure (WBS). A WBS is a product-oriented family-tree subdivision of the hardware, services and data required for project realization, which provides a common framework for breaking the work down into work packages, thus providing a greater probability that every major and minor activity will be accounted for (Kerzner 1997).

In our model, each project $k$ consists of an aggregated workload on a bottleneck resource, expressed as a discrete number $p_{k}$ of work packages. Precedence relations and nonpreemption constraints may apply between the work packages, for more details we refer to Section 3.4. An accepted order can only be executed between its release time $r_{k}$ (a full description of the arrival process is given in Section 3.2 and the project's due date $d_{k}$, which is regarded here as a deadline. This implies that due dates cannot be exceeded and thus orders for which the due date cannot be met, must be rejected. The payoff of a work order, denoted as $y_{k}$, is generated immediately when the work is completed. We assume that all these revenues can be reinvested at a fixed interest rate $i>0$.

\subsection{The order arrival process}

A Request for Proposal (referred to as RFP) is an invitation for suppliers, through a bidding process, to bid on a specific product or service. An RFP typically involves more than the price, which is why, in the context of this article, it may be more appropriate to use the term Request for Quotation (RFQ), where discussions are not required with bidders (mainly when the specifications of a product or service are already known), and price is the main or only factor in selecting the successful bidder. In what follows, we use the terms 'order' and 'project' to refer either to an RFQ or to a request for execution of an order at a given price. Since we establish a profit threshold below which prices lead to rejection, price setting and order acceptance at a fixed price can be treated similarly. Our models are developed 


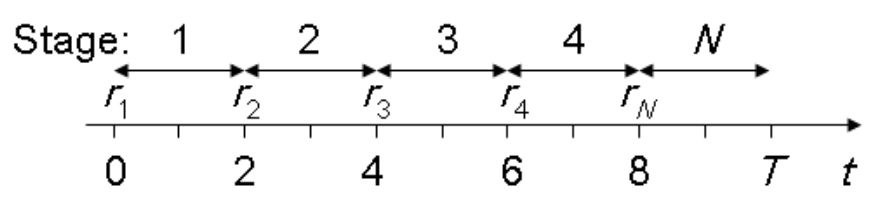

Figure 1: Order arrival process

from the viewpoint of one individual bidder, and decisions are made without consideration of competitors.

The stream of oncoming order arrivals is the main source of uncertainty in dynamic order acceptance. When a company has to make an accept/reject decision, it has at its disposal only rudimentary information about the project in question and forecasts of the main characteristics of the future incoming projects (e.g. based on sales-force polling). In the following paragraphs we describe the assumptions underlying our model of the order arrival process.

We discretize the planning horizon into $T$ periods or time buckets (e.g. days or weeks). Additionally, we introduce the concept of a stage, which is the time interval between two consecutive project arrivals: a new stage starts every time a new project arrives. The number of projects arriving sequentially within the planning horizon $T$ (and hence, the number of stages) is $N$. We assume that the arrivals have equal interarrival times. A visualization of the order arrival process is given in Figure 1 with $T=10$ and $N=5$.

In Section 3.1 it was explained that the main characteristics of a project proposal $k$ are its payoff $y_{k}$, a positive workload $p_{k}$ and a due date $d_{k}$. From a given positive maximal time lag $l_{k}$ allowed for realizing the project, one can easily derive the due date by adding the stage's release time $r_{k}$ to the lead time: $d_{k}=r_{k}+l_{k}$. We represent the arriving offers as $\mathbf{w}_{1}, \mathbf{w}_{2}, \ldots, \mathbf{w}_{N}$, with $\mathbf{w}_{k}=\left(p_{k}, y_{k}, l_{k}\right)$, for stage $k=1, \ldots, N$. A decision needs to be made regarding order $\mathbf{w}_{1}$, and estimates about the characteristics of the stream of future order arrivals are captured as follows: values $p_{k}, y_{k}$ and $l_{k}$ are assumed to be realizations of $P, Y$ and $L$, respectively, each of which is a random variable (r.v.). Hence, the values $\mathbf{w}_{k}$, $k=2, \ldots, N$, are independent realizations of multivariate r.v. $\mathbf{W}=(P, Y, L)$. The support of $P$ and $L$ only contains natural numbers.

At the start of each stage $k$, we decide upon order acceptance and capacity allocation of project $k$, with stage 1 being the first stage. Project $k$ can be planned from its release time $r_{k}$, which is in fact the start of stage $k$. It will turn out in Section 4 that there is a 
one-to-one correspondence between the stages in the order arrival process and the stages of the SDP algorithms. Our assumption of equal interarrival times seems restrictive, but is a representation of the fact that we use forecasts of the future arrival stream, for which only an average interarrival time is known. When this leads to non-discrete time instants, the planning decisions for each project are shifted towards the start of the next period. Varying time intervals are easily incorporated, but would not really add to the value of the results of the current text. Stochastic interarrival times can be modelled by appropriately increasing the number of stages within a fixed time horizon and adapting the distribution of $Y$ (event $Y=0$ then corresponds with no arrival).

\subsection{Capacity profile}

In this paper we consider only one resource type, which is taken to represent the bottleneck resource of the company. For R\&D projects, for instance, this resource could be a critical testing equipment or the allocated periodical budget, while in an MTO environment it might represent a single machine or a team of engineers. The company owns a limited number of bottleneck capacity units. The amount of regular capacity units is the result of a long-term strategic decision that cannot be revised within the time horizon considered in our planning framework. In contrast, the amount of non-regular capacity units can be altered as a result of working overtime, hiring temporary labor or outsourcing.

We count the available regular and non-regular capacity units in every time period by means of a capacity profile, which is a vector

$$
\mathbf{x}_{k}=\left(\mathbf{m}_{k}, \mathbf{s}_{k}\right), \text { with }\left\{\begin{array}{l}
\mathbf{m}_{k}=\left(m_{r_{k}}, m_{r_{k}+1}, \ldots, m_{T}\right) \\
\mathbf{s}_{k}=\left(s_{r_{k}}, s_{r_{k}+1}, \ldots, s_{T}\right)
\end{array}\right.
$$

where $k$ represents the stage number. Here $m_{t}$ is the number of available regular capacity units in time period $t$ and $s_{t}$ the maximum number of non-regular capacity units that can be hired during time period $t$. The cost per unit of consumed non-regular capacity is $c$, whereas the actual utilization of regular capacity does not give rise to incremental costs. In stage $k, \mathbf{x}_{k}$ only reflects resource availability from time $r_{k}$ onwards since this vector contains all information relevant for making decisions regarding offer $k$. Implicitly, this derives from the fact that all unused capacity units before $r_{k}$ have 'perished'. In the remainder of this article, we will speak of perishable resources (cfr. Weatherford \& Bodily 1992); the scheduling literature sometimes uses the term renewable resources (see, for instance, Demeulemeester \& Herroelen 2002). 


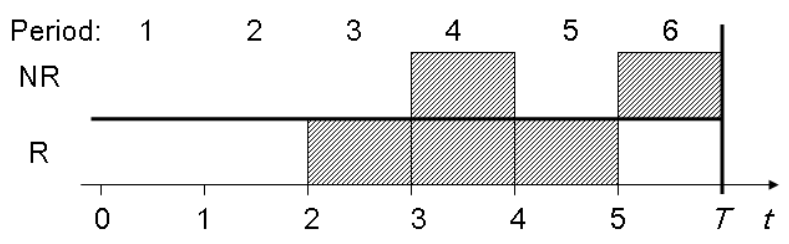

Figure 2: Capacity profile with regular (R) and non-regular (NR) capacity units

An illustration is provided in Figure 2. The capacity profile for the first stage, starting at time 0 , is $\mathbf{x}_{1}=\left(\mathbf{m}_{1}, \mathbf{s}_{1}\right)=((0,0,1,1,1,0),(0,0,0,1,0,1))$. Remark that even the capacity profile in stage 1 can exhibit an uneven pattern: earlier decisions, e.g. under the form of firm planned orders, may already have allocated capacity units in the current planning horizon.

\subsection{The order plan}

Upon arrival, the organization can choose whether to reject or to accept the project according to any eligible order plan. An order plan is an allocation of capacity units to the different work packages of a project. We represent an order plan $j$ for arrival $k$ as a vector $\mathbf{a}_{k}^{j}=$ $\left(\mathbf{a}_{k m}^{j}, \mathbf{a}_{k s}^{j}\right)$, where $\mathbf{a}_{k m}^{j}$ and $\mathbf{a}_{k s}^{j}$ have the same dimension as $\mathbf{m}_{k}$ and $\mathbf{s}_{k}$, and count the number of (regular and non-regular) capacity units that are allocated to project $k$ in each relevant time period.

We define an order plan to be feasible if two conditions are fulfilled: (1) the total workload of the project is covered; and (2) all work packages are planned between the stage's release time $r_{k}$ and the project's deadline $d_{k}$. The set of feasible order plans in stage $k$ is denoted as $\mathcal{F}_{k}$. Additionally, the set of order plans to be examined may be reduced because of practical considerations, which may take the form of precedence relations between the work packages or non-preemption constraints. It may be the case, for instance, that a complete order is imposed on the work packages (e.g. stage-gate development processes, see Cooper et al. 1999), or that sequential testing procedures need to take place in consecutive time periods.

When an offer is accepted according to a specific order plan, the corresponding capacity allocation is immediately locked: the acceptance and planning decisions are made simultaneously. We do not allow for replanning the project: once an order plan is selected, it cannot be altered. In case the company rejects the offer, no capacity is reserved and no further action is taken until the next project arrival. This rejection decision cannot be withdrawn. 


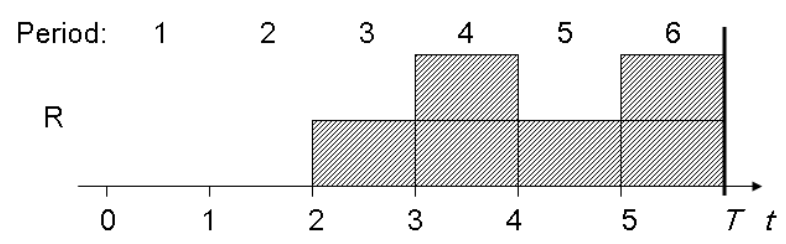

Figure 3: Capacity profile with regular (R) capacity units

We associate a 'degenerate' order plan $\mathbf{a}_{k}^{0}=\mathbf{0}$ (the null vector) with rejection, and we let symbol $\mathcal{A}_{k}$ represent the set of all eligible order plans augmented with $\mathbf{a}_{k}^{0}$.

\subsection{A small example}

Consider an MTO organization with the Testing Department containing two fabrication lines as a bottleneck; the duration of a time bucket is one week. In Figure 3 the current capacity profile is shown: $\mathbf{m}_{1}=(0,0,1,2,1,2)$ and we do not consider overtime opportunities $\left(\mathbf{s}_{1}=\mathbf{0}\right)$. The company has just received an RFQ for a project with a deadline six weeks from now $\left(l_{1}=6\right)$. The company estimates that the execution of the order would require three regular capacity units: $p_{1}=3$. Due to very large set-up times, preemption is not beneficial. The company has drawn up the set of eligible order plans $\mathbf{a}_{1 m}^{j}, j=1, \ldots, 5$, for project proposal $\mathbf{w}_{1}$. They are given below:

$$
\begin{array}{ll}
\mathbf{a}_{1 m}^{1}=(0,0,1,2,0,0) & \mathbf{a}_{1 m}^{2}=(0,0,1,1,1,0) \\
\mathbf{a}_{1 m}^{4}=(0,0,0,1,1,1) & \mathbf{a}_{1 m}^{3}=(0,0,0,2,1,0) \\
\mathbf{b}_{1 m}^{5}=(0,0,0,0,1,2)
\end{array}
$$

For instance, the first order plan represents a capacity allocation of one unit from period 3 and two units from period 4 to the incoming project.

The marketing department has forecast the incoming order arrival stream as being composed of two types of projects, some $30 \%$ of which belong to the first type. The average characteristics of each of these two types are described by $\mathbf{W}=(P, Y, L)$, with the following probabilities:

$$
\operatorname{Pr}[(1,5,3)]=0.3, \quad \operatorname{Pr}[(2,8,4)]=0.7 .
$$

Marketing foresees an arrival rate of one incoming order per week over the planning horizon of 6 weeks $(N=6)$. Thus, for this example, every stage corresponds to one time period.

In the next section we develop an SDP method to determine the minimal price to be quoted for an RFQ and to pick the best order plan. 


\section{Stochastic dynamic programming}

We present an SDP approach (see, for instance, Ross 1983) for order acceptance and capacity planning. The problem is modelled as an extension of the optimal stopping problem (e.g. Bertsekas 2005) for which we regard the capacity units as perishable assets. By selling the assets, individually or in group, at the highest expected offer, we maximize the expected profits. This interpretation of the problem will allow us to determine an optimal threshold and order plan for every arriving offer.

The basic optimal stopping problem is discussed in Section 4.1, our extensions are presented in the following subsections. Our first model, presented in Section 4.2, only considers project proposals with single-sized workloads and infinite deadlines. A more general model is elaborated in Section 4.3 ,

\subsection{Optimal stopping problem}

We investigate and extend one specific variant of the optimal stopping problem, namely the asset selling problem as described in Bertsekas (2005). In this setting, an asset seller receives a random bid $w_{k}$ in each period, over a horizon of $N$ periods. If the person accepts the offer, he or she receives the payoff which can be reinvested at an interest rate $i$.

Bertsekas presents an SDP to determine an optimal threshold in every period for accepting a bid. Its elements are the following: the state of the system in each stage $k$, represented by $x_{k}$, and the control space. If the bid is accepted, the system goes into the termination state $T$, otherwise the state equals the last considered bid. The control space contains the possible actions we can undertake when arriving in a new stage, which is at the arrival of a new bid. There are two possible actions $a_{k}$, namely $a_{k}^{0}$ : the rejection of the bid, and $a_{k}^{1}$ : the selling of the asset.

\subsection{Dynamic order acceptance and planning for single-sized orders}

Based on the previously discussed optimal stopping problem, we develop an SDP consisting of $N$ stages, where $N$ equals the number of offers within the planning horizon $T$, as was described in Section 3.2. The arrival of an order corresponds to the beginning of a new stage $k$. The state in stage $k$ is the capacity profile $\mathbf{x}_{k}$. To alleviate the notation, we omit the perished periods from the state vector (which was also suggested in Section 3.3). To this aim we define a perishing function $v$ that transforms a vector $\mathbf{x}_{k}$ into a vector $\mathbf{x}_{k+1}$ from 
which the perished capacity units are removed. For the example in Section 3.5, we have $v\left(\mathbf{x}_{3}\right)=\mathbf{x}_{4}=((2,1,2),(0,0,0))$. We call a specific capacity unit perishing if it perishes in the following stage.

The control space $\mathcal{A}_{k}$ of the SDP consists of a variable number of possible actions, each corresponding with an order plan $\mathbf{a}_{k}^{j}$. The reward in stage $k$ for order plan $\mathbf{a}_{k}^{j}$ and offer $\mathbf{w}_{k}$ is determined as:

$$
g_{k}\left(\mathbf{a}_{k}^{j}, \mathbf{w}_{k}\right)= \begin{cases}0 & \text { if } j=0, \\ y_{k}(1+i)^{\left(T-t_{j}^{*}\right)}-c s_{j}^{*} & \text { otherwise, }\end{cases}
$$

where $s_{j}^{*}$ is equal to 1 if order plan $\mathbf{a}_{k}^{j}$ allocates a non-regular capacity unit, and equal to 0 otherwise. $t_{j}^{*}$ refers to the realization time of an accepted project which is planned according to $\mathbf{a}_{k}^{j}$. This point in time corresponds with the pay-out time of the project. As a result, $T-t_{j}^{*}$ is the period for which the company receives additional interest revenues.

The backward SDP algorithm consists of iteratively solving the following recursion:

$$
\left\{\begin{aligned}
f_{N}\left(\mathbf{x}_{N}\right) & =\max _{\mathbf{a}_{N}^{j} \in \mathcal{A}_{N}}\left\{g_{N}\left(\mathbf{a}_{N}^{j}, \mathbf{w}_{N}\right)\right\} & \text { if } k=N, \\
f_{k}\left(\mathbf{x}_{k}\right) & =\max _{\mathbf{a}_{k}^{j} \in \mathcal{A}_{k}}\left\{g_{k}\left(\mathbf{a}_{k}^{j}, \mathbf{w}_{k}\right)+E\left[f_{k+1}\left(\mathbf{x}_{k+1}\right)\right]\right\} & \text { if } k \neq N,
\end{aligned}\right.
$$

with

$$
\mathbf{x}_{k+1}=v\left(\mathbf{x}_{k}-\mathbf{a}_{k}^{j}\right)
$$

and $E[\cdot]$ the expectation operator. $\mathbf{x}_{k+1}$ represents the state or capacity profile after implementing order plan $\mathbf{a}_{k}^{j}$; if $j=0, \mathbf{x}_{k+1}$ equals $v\left(\mathbf{x}_{k}\right)$.

In Eq. (3), $f_{k}\left(\mathbf{x}_{k}\right)$ is the maximum expected reward that can be earned during stages $k, k+1, \ldots, N$ given that the initial state corresponds with $\mathbf{x}_{k}$.

Definition 1. In stage $k$, an order plan $\mathbf{a}_{k}^{\tilde{j}}$ is dominated by another order plan $\mathbf{a}_{k}^{j}$ if

$$
\begin{array}{rlrl}
g_{N}\left(\mathbf{a}_{N}^{j}, \mathbf{w}_{N}\right) & \geq g_{N}\left(\mathbf{a}_{N}^{\tilde{j}}, \mathbf{w}_{N}\right) & & \text { if } k=N, \\
g_{k}\left(\mathbf{a}_{k}^{j}, \mathbf{w}_{k}\right)+E\left[f_{k+1}\left(\mathbf{x}_{k+1}\right)\right] \geq g_{k}\left(\mathbf{a}_{k}^{\tilde{j}}, \mathbf{w}_{k}\right)+E\left[f_{k+1}\left(\tilde{\mathbf{x}}_{k+1}\right)\right] & & \text { if } k \neq N .
\end{array}
$$

Intuitively, one order plan dominates another if the second does not result in a larger value for $f_{k}\left(\mathbf{x}_{k}\right)$ according to Eq. (3). Theorems 1, 2 and 3 below describe a number of situations in which dominated order plans can be recognized.

Theorem 1. An order plan which employs non-regular capacity units that can be replaced or partially replaced by regular capacity units from the same time period is always dominated by an order plan which has adopted these replacements. 
All proofs appear in the appendix.

Definition 2. A state $\mathbf{x}_{k}$ is larger than $\left(>_{l}\right)$ another state $\tilde{\mathbf{x}}_{k}$ if the capacity profile of $\mathbf{x}_{k}$ contains every capacity unit of the capacity profile of $\tilde{\mathbf{x}}_{k}$ and at least one additional capacity unit. Two states are equal (=) if their stage number and capacity profile are the same.

Lemma 1. If $\mathbf{x}_{k}$ is larger than $\left(>_{l}\right) \tilde{\mathbf{x}}_{k}$ then the maximum expected reward from $\mathbf{x}_{k}$ is larger than or equal to the maximum expected reward from $\tilde{\mathbf{x}}_{k}$.

Theorem 2. An order plan that results in a non-positive reward is always dominated by rejecting the offer.

Theorem 3. An order plan $\mathbf{a}_{k}^{j}$ that employs one or more perishing regular capacity units dominates any order plan $\mathbf{a}_{k}^{\tilde{j}}$ that has replaced one or more of these perishing regular capacity units with non-perishing capacity units.

Under certain conditions, the theorem can be strengthened.

Corollary 1. When $\mathcal{A}_{k}=\mathcal{F}_{k} \cup\left\{\mathbf{a}_{k}^{0}\right\}$, any order plan that allocates non-perishing capacity units while leaving perishing regular capacity units unallocated, is dominated.

By iterative solution of the SDP recursion of Eq. (3) we can derive the optimal acceptance threshold $\alpha_{k}$ and the optimal planning method for incoming order $\mathbf{w}_{k}$. To this aim we define $f_{k}^{j}\left(\mathbf{x}_{k}\right)$ as $g_{k}\left(\mathbf{a}_{k}^{j}, \mathbf{w}_{k}\right)+E\left[v\left(\mathbf{x}_{k+1}-\mathbf{a}_{k}^{j}\right)\right]$ if $k \neq N$ and as $g_{N}\left(\mathbf{a}_{N}^{j}, \mathbf{w}_{k}\right)$ if $k=N . f_{k}^{j}\left(\mathbf{x}_{k}\right)$ has slope 0 if $j=0$ and slope $(1+i)^{\left(T-t_{j}^{*}\right)} \geq 1$ otherwise. The non-decreasing piecewise linear function $f_{k}\left(\mathbf{x}_{k}\right)$ can now be written as $\max _{\mathbf{a}_{k}^{j} \in \mathcal{A}_{k}}\left\{f_{k}^{j}\left(\mathbf{x}_{k}\right)\right\}$. In each of the subintervals $\left[0, \alpha_{k}\left[,\left[\alpha_{k}, \theta_{k}^{1}\left[,\left[\theta_{k}^{1}, \theta_{k}^{2}\left[, \ldots,\left[\theta_{k}^{n}, \infty\left[\right.\right.\right.\right.\right.\right.\right.\right.$ of $y_{k}$, the function $f_{k}\left(\mathbf{x}_{k}\right)$ coincides with one of the functions $f_{k}^{j}\left(\mathbf{x}_{k}\right)$. The threshold $\alpha_{k}$ is $\max \left\{y_{k} \mid f_{k}^{0}\left(\mathbf{x}_{k}\right) \geq f_{k}^{j}\left(\mathbf{x}_{k}\right), j \neq 0\right\}$. For each subinterval, the best order plan is the one that maximizes $f_{k}\left(\mathbf{x}_{k}\right)$ in that interval, as visualized in Figure 4 .

Observation 1. The maximum expected reward that can be earned during stages $k, k+$ $1, \ldots, N$ for a given initial state $\mathbf{x}_{k}, f_{k}\left(\mathbf{x}_{k}\right)$, is a non-decreasing convex function of the current project payoff $y_{k}$.

To illustrate the solution method we consider a company that has been offered a project with a payoff of 10 if realized with regular capacity $\left(y_{1}=10\right)$. Assume we are at time instant 0 looking at the next three weeks $(T=3)$. The capacity profile within the problem horizon 


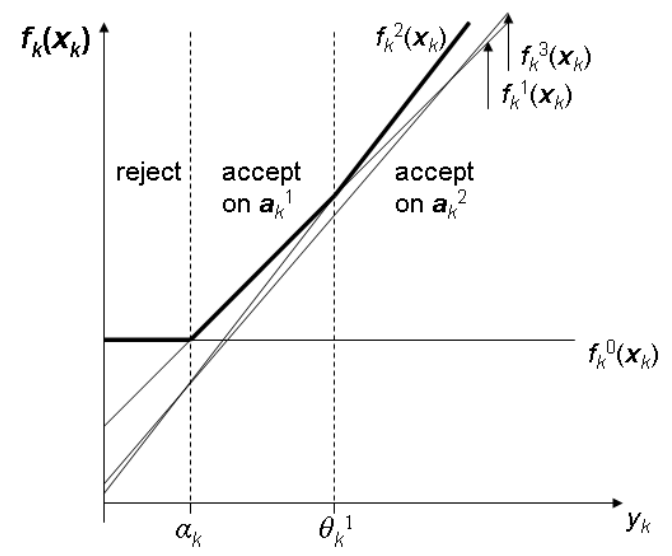

Figure 4: Threshold and order plan determination

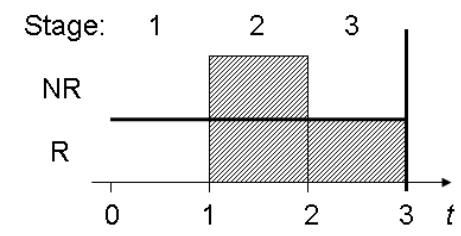

Figure 5: Example with regular (R) and non-regular (NR) capacity units

is $\mathbf{x}_{1}=((0,1,1),(0,1,0))$ and is depicted in Figure 5. We ignore the possibility to reinvest the revenues and set the interest rate $i$ to 0 . The cost of one unit of non-regular capacity is $c=5$. There is one project arrival per week $(N=3)$ and the payoffs of the incoming orders are forecast to adhere to a continuous uniform distribution, with probability distribution function:

$$
\begin{aligned}
u_{Y}(y) & =\frac{1}{12}, & 2 \leq y \leq 14, \\
& =0, & \text { otherwise. }
\end{aligned}
$$

The DP algorithm described by Eq. (3) corresponds with the third-stage values, $(k=N)$ :

$$
\begin{array}{ll}
f_{3}((1),(0)) & =y_{3}, \\
E\left[f_{3}((1),(0))\right] & =\int_{2}^{14}\left(\frac{1}{12} y\right) d y=8 \\
f_{3}((0),(0)) & =0
\end{array}
$$

For $f_{3}((1),(0))$, we can choose between two order plans: $\mathbf{a}_{3}^{0}$ and $\mathbf{a}_{3}^{1}$. $\mathbf{a}_{3}^{0}$ is the null vector: $\mathbf{0}_{3}=((0),(0))$. Order plan $\mathbf{a}_{3}^{1}=((1),(0))$ implies the allocation of the regular capacity unit from period 3 to the project. Since rejection has a reward of 0 , order plan $\mathbf{a}_{3}^{1}$ maximizes the reward.

The values for stage 2 are the following. We only show the state vectors that will be employed 
in the first stage, and we identify the elements of the control space between straight brackets:

$$
\begin{array}{rll}
f_{2}((1,1),(1,0)) & =\max \left\{\begin{array}{ll}
E\left[f_{3}((1),(0))\right] & {\left[\mathbf{a}_{2}^{0}=\mathbf{0}_{2}\right]} \\
y_{2}+E\left[f_{3}((1),(0))\right] & {\left[\mathbf{a}_{2}^{1}=((1,0),(0,0))\right]} \\
y_{2}+E\left[f_{3}((0),(0))\right] & {\left[\mathbf{a}_{2}^{2}=((0,1),(0,0))\right]}
\end{array}\right\}, \\
E\left[f_{2}((1,1),(1,0))\right] & =y_{2}+E\left[f_{3}((1),(0))\right], \\
f_{2}((0,1),(1,0)) & =\max \left\{E\left[f_{3}((1),(0))\right] ; y_{2}-5+E\left[f_{3}((1),(0))\right] ; y_{2}\right\}, \\
E\left[f_{2}((0,1),(1,0))\right] & =\int_{2}^{5}\left(\frac{1}{12} \cdot 8\right) d y+\int_{5}^{14} \frac{1}{12}(y+3) d y=11.5, \\
f_{2}((1,0),(1,0)) & =y_{2}, \\
E\left[f_{2}((1,0),(1,0))\right] & =\int_{2}^{14}\left(\frac{1}{12} y\right) d y=8 .
\end{array}
$$

With $f_{2}((1,1),(1,0))$, we associate three eligible order plans: $\mathbf{a}_{2}^{0}$, $\mathbf{a}_{2}^{1}$ and $\mathbf{a}_{2}^{2}$. Following Theorem 3, we need not consider the last order plan $\mathbf{a}_{2}^{2}$ since it is dominated by $\mathbf{a}_{2}^{1}$. The best order plan for $f_{2}((0,1),(1,0))$ cannot be determined unambiguously. If the payoff of the project proposal lies within the interval $[2,5]$, rejection maximizes the expected value of the following stages. If the income is within [5, 14], order plan $\mathbf{a}_{2}^{1}=((0,0),(1,0))$ becomes the best choice.

In the first stage, we have:

$$
\begin{aligned}
f_{1}((0,1,1),(0,1,0)) & =\max \left\{\begin{array}{ll}
E\left[f_{2}((1,1),(1,0))\right] & {\left[\mathbf{a}_{1}^{0}=\mathbf{0}\right]} \\
E\left[f_{2}((0,1),(1,0))\right]+10 & {\left[\mathbf{a}_{1}^{1}=((0,1,0),(0,0,0))\right]} \\
E\left[f_{2}((1,0),(1,0))\right]+10 & {\left[\mathbf{a}_{1}^{2}=((0,0,1),(0,0,0))\right]}
\end{array}\right\}, \\
& =\max \{16 ; 21.5 ; 18\} .
\end{aligned}
$$

In combination with the example's capacity profile, we can choose from three order plans in the first stage. The first corresponds to rejection; the other two consist of planning in period 2 and 3 , respectively. Since $E\left[f_{2}((0,1),(1,0))\right]>E\left[f_{2}((1,0),(1,0))\right]$, the optimal payoff threshold for accepting a project at time 0 is $\alpha_{1}=4.5$. The incoming order will preferably be planned in time period 2 .

\subsection{General dynamic order acceptance and planning}

In this section, we generalize the solution method for orders with different workloads and due dates. The overall solution approach is the same as in the previous section but the implementation becomes a bit more intricate.

For the reward $g_{k}$ in stage $k$, a redefinition of $s_{j}^{*}$ is due: $s_{j}^{*}$ now denotes the number of non-regular capacity units employed when implementing order plan $\mathbf{a}_{k}^{j}$. Theorems 1, 2, and 3 remain valid. The SDP recursion from Eq. (3) can be used to solve the generalized problem. 


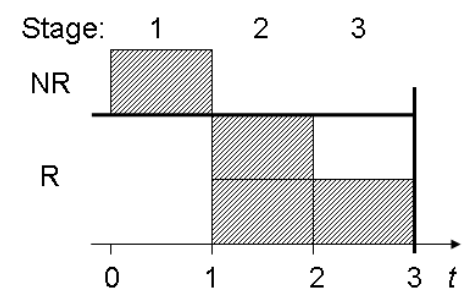

Figure 6: Example with regular (R) and non-regular (NR) capacity units

The method for the calculation of the optimal acceptance threshold and the derivation of the best order plan as given in previous section (Section 4.2), remains valid.

As an illustration, we consider a project offered to a company at time 0 . The company assesses that this project would generate a payoff of $18\left(=y_{1}\right)$ if realized with regular capacity. The project would require two work packages of the company's key resource $\left(p_{1}=2\right)$. Delivery of the project is due within two weeks $\left(l_{1}=2\right)$. The problem horizon $T$ is set to three weeks. At this moment, the profile of the available capacity is $\mathbf{x}_{1}=((0,2,1),(1,0,0))$, a visualization is given in Figure 6. The interest rate $i$ is set to $10 \%$ and the cost of one unit of non-regular capacity amounts to 10 . We anticipate the arrival of one RFQ per week within the problem horizon, so that $N=3$. The company has defined six order types, the average characteristics of which are described by $\mathbf{W}=(P, Y, L)$. The forecasting information contains the following probabilities:

$$
\begin{array}{lll}
\operatorname{Pr}[(1,8,1)]=0.1, & \operatorname{Pr}[(1,8,2)]=0.1, \\
\operatorname{Pr}[(1,12,1)]=0.2, & & \operatorname{Pr}[(1,12,2)]=0.1, \\
\operatorname{Pr}[(2,18,1)]=0.1, & & \operatorname{Pr}[(2,18,2)]=0.4 .
\end{array}
$$

We derive the following third-stage values:

$$
\begin{aligned}
& f_{3}((1),(0))= \begin{cases}y_{3}, & \text { if } p_{3}=1, \\
0, & \text { if } p_{3}=2,\end{cases} \\
& E\left[f_{3}((1),(0))\right]=0.1(8+8+12)+0.2 \times 12+0.1 \times 0+0.4 \times 0=5.2, \\
& f_{3}((0),(0))=0 .
\end{aligned}
$$

We calculate $E\left[f_{3}((1),(0))\right]$ as the sum of the probability of the different order types multiplied with the corresponding value for $f_{3}((1),(0))$. 
The second-stage computations are:

$$
\begin{aligned}
& f_{2}((2,1),(0,0))=y_{2}(1.1)+E\left[f_{3}((1),(0))\right] \\
& E\left[f_{2}((2,1),(0,0))\right]=14.2 \times 1.1+5.2=20.82 \text {, } \\
& f_{2}((2,0),(0,0))=y_{2}(1.1)+E\left[f_{3}((1),(0))\right] \text {, } \\
& E\left[f_{2}((2,0),(0,0))\right]=20.82 \\
& f_{2}((1,1),(0,0))= \begin{cases}y_{2}(1.1)+E\left[f_{3}((1),(0))\right], & \text { if } p_{2}=1, \\
\max \left\{E\left[f_{3}((1),(0))\right] ; y_{2}\right\}, & \text { if } p_{2}=2 \wedge l_{2}=2 \\
0, & \text { otherwise }\end{cases} \\
& E\left[f_{2}((1,1),(0,0))\right]=0.2(8 \times 1.1+5.2)+0.3(12 \times 1.1+5.2)+0.4 \times 18=15.52 \text {, } \\
& f_{2}((1,0),(0,0))= \begin{cases}y_{2}(1.1)+E\left[f_{3}((1),(0))\right], & \text { if } p_{2}=1 \\
0, & \text { otherwise }\end{cases} \\
& E\left[f_{2}((1,0),(0,0))\right]=0.2(8 \times 1.1+5.2)+0.3(12 \times 1.1+5.2)=8.32, \\
& f_{2}((0,1),(0,0))= \begin{cases}\max \left\{E\left[f_{3}((1),(0))\right] ; y_{2}\right\}, & \text { if } p_{2}=1 \wedge l_{2}=2, \\
0, & \text { otherwise, }\end{cases} \\
& E\left[f_{2}((0,1),(0,0))\right]=0.1 \times 8+0.1 \times 12=2 \text {, } \\
& f_{2}((0,0),(0,0))=0 \text {. }
\end{aligned}
$$

The first-stage computations suggest acceptance of the project $\mathbf{w}_{1}$ :

$$
\begin{aligned}
f_{1}((0,2,1),(1,0,0)) & =\max \left\{\begin{array}{ll}
E\left[f_{2}((2,1),(0,0))\right] & {\left[\mathbf{a}_{1}^{0}\right]} \\
E\left[f_{2}((1,1),(0,0))\right]+18 \times 1.1-10 & {\left[\mathbf{a}_{1}^{1}\right]} \\
E\left[f_{2}((0,1),(0,0))\right]+18 \times 1.1 & {\left[\mathbf{a}_{1}^{2}\right]}
\end{array}\right\}, \\
& =\max \{20.82 ; 25.32 ; 21.8\}
\end{aligned}
$$

In stage one, three order plans are taken into consideration; the first one equals $((0,0,0),(0,0,0))$. The second order plan $((0,1,0),(1,0,0))$ allocates the non-regular capacity unit from period 1 at a cost of 10 and the regular capacity unit from period 2. The finish time of the project precedes the end of the planning horizon, $T$, so that interest revenues are reaped. The third possibility $((0,2,0),(0,0,0))$ is to plan on the two regular capacity units from the second period, so that the finish time of this order plan is the same as of the first plan. From the stage-one computations, we learn that the order can be optimally executed with one nonregular capacity unit from period 1 and one regular capacity unit from the second period. In addition, we formulate a payoff threshold as an acceptance rule for incoming projects with the same characteristics. For this example the required minimal payoff is 13.91 in combination with order plan $\mathbf{a}_{1}^{1}$. However, if we changed the cost $c$ of a unit non-regular capacity to 15 , it would no longer be optimal to make use of this capacity unit. In this case, $f_{1}((0,2,1),(1,0,0))=\max \{20.82 ; 20.32 ; 21.8\}$, so that the best order plan would be $\mathbf{a}_{1}^{2}$ which uses the free capacity from period 2. In addition, the payoff threshold would be raised to 17.11 . 


\section{Insights and discussion}

In Section 4 we have derived project-specific acceptance thresholds which equal the minimal revenue desired from a project. For the optimal stopping problem (Section 4.1), on the other hand, the threshold is actually the minimal bid required for the sale of one specific capacity unit. While elaborating this basic model, we have in fact determined minimal prices for specific combinations of capacity units as specified by the order plans. The best combination of capacity units led us to a payoff threshold for the incoming project. Based on the threshold calculation in Section 4.2 (and contrary to the models of Bertsekas), one can easily construct examples where the threshold for a specific project exhibits an irregular evolution in the stage number.

The SDP recursion in Eq. (3) shows that optimal acceptance and planning decisions depend on (1) the immediate reward and (2) the expected future rewards. The immediate reward can easily be maximized, while high expected future rewards are the result of a good fit between the future arrival characteristics and the capacity profile (after the stage's capacity allocation). Unfortunately, optimal 'short-cut' rules cannot easily be determined, and may not even exist, since the influential characteristics have diverging effects. For example, different properties of the order arrival stream (e.g. finite maximal time lags) lead to better results in combination with different capacity profiles (e.g. levelled profile).

Using a general framework, we isolate and quantify the different influential effects. In our analysis we determine the specific circumstances under which 'simple' planning rules are optimal. In the remainder of this section, we first present the general framework that will be used to examine the influential characteristics. Subsequently, we separate and quantify the effects from perishable capacity units, finite time lags and reinvestment revenues. Finally, we estimate the worth of having a non-regular capacity unit at one's disposal and we quantify the value of due-date flexibility.

\subsection{General framework}

We consider a general framework with single-sized incoming projects of order type $A$ and $B$. Order type $z$ has a maximal time lag of $l_{z}$ and a payoff of $y_{z}, z=A, B$; we assume that $y_{A}>y_{B}$. The probability that an arrival belongs to type $z$ is denoted as $\operatorname{Pr}[z]$. We consider the capacity profile $\mathbf{x}_{1}=((0, \ldots, 1,1),(0, \ldots, 0,1))$. Within our framework, periods coincide

with stages. The cost of a non-regular capacity unit may vary between 0 and $\infty$, and we 
distinguish three cases:

(a) $c=0$ : the available overtime is free;

(b) $0<c<\infty$ : overtime can be hired at a certain cost;

(c) $c=\infty$ : there are no overtime opportunities.

These three cases are visualized in Figure 7.

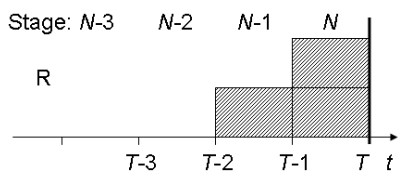

(a) $c=0$

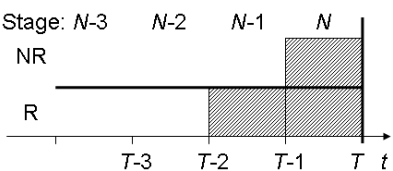

(b) $c>0$

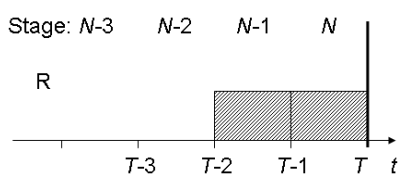

(c) $c=\infty$

Figure 7: General framework with regular (R) and non-regular (NR) capacity units

The stage- $N$ values are derived for the general case:

$$
\begin{aligned}
& E\left[f_{N}((1),(1))\right]=\operatorname{Pr}[A] \cdot y_{A}+\operatorname{Pr}[B] \cdot y_{B}=\bar{y} \\
& E\left[f_{N}((0),(1))\right]=\operatorname{Pr}[A] \cdot \max \left\{y_{A}-c ; 0\right\}+\operatorname{Pr}[B] \cdot \max \left\{y_{B}-c ; 0\right\} .
\end{aligned}
$$

Using the recursion from Eq. (3) and invoking Theorem 1 we obtain the expected values for stage $N-1$ :

$$
\begin{aligned}
E\left[f_{N-1}((1,1),(0,1))\right]= & \bar{y}(2+i), \\
E\left[f_{N-1}((1,0),(0,1))\right]= & \bar{y}(1+i)+\sum_{z=A, B} \operatorname{Pr}[z] \cdot \max \left\{y_{z}-c ; 0\right\}, \\
E\left[f_{N-1}((1,0),(0,0))\right]= & \bar{y}(1+i), \\
E\left[f_{N-1}((0,1),(0,1))\right]= & \sum_{z=A, B} \operatorname{Pr}[z] \cdot \max \left\{\text { if } l_{z}>1: y_{z}+E\left[f_{N}((0),(1))\right], \text { else } 0 ; \bar{y}\right\}, \\
E\left[f_{N-1}((0,0),(0,1))\right]= & \operatorname{Pr}[A] \cdot \max \left\{\text { if } l_{A}>1: y_{A}-c, \text { else } 0 ; \operatorname{Pr}[A] \cdot \max \left\{y_{A}-c ; 0\right\}\right. \\
& \left.+\operatorname{Pr}[B] \cdot \max \left\{y_{B}-c ; 0\right\}\right\}+\operatorname{Pr}[B] \cdot\left(\operatorname{Pr}[A] \cdot \max \left\{y_{A}-c ; 0\right\}+\right. \\
& \left.\operatorname{Pr}[B] \cdot \max \left\{y_{B}-c ; 0\right\}\right) .
\end{aligned}
$$

For stage $N-2$, we calculate:

$$
\begin{aligned}
E\left[f_{N-2}((0,1,1),(0,0,1))\right]= & \sum_{z=A, B} \operatorname{Pr}[z] \cdot \max \left\{\text { if } l_{z}>2: y_{z}+E\left[f_{N-1}((1,0),(0,1))\right],\right. \\
& \text { else } 0 ; \text { if } l_{z}>1: y_{z}(1+i)+E\left[f_{N-1}((0,1),(0,1))\right], \text { else 0; } \\
& \bar{y}(2+i)\}, \\
E\left[f_{N-2}((0,1,0),(0,0,1))\right]= & \sum_{z=A, B} \operatorname{Pr}[z] \cdot \max \left\{\text { if } l_{z}>2: y_{z}-c+\right. \\
& E\left[f_{N-1}((1,0),(0,0))\right] \text { else } 0 ; \text { if } l_{z}>1: y_{z}(1+i) \\
& \left.+E\left[f_{N-1}((0,0),(0,1))\right], \text { else } 0 ; E\left[f_{N-1}((1,0),(0,1))\right]\right\}, \\
E\left[f_{N-2}((0,0,1),(0,0,1))\right]= & \sum_{z=A, B} \operatorname{Pr}[z] \cdot \max \left\{\text { if } l_{z}>2: y_{z}+E\left[f_{N-1}((0,0),(0,1))\right],\right. \\
& \text { else } \left.0 ; E\left[f_{N-1}((0,1),(0,1))\right]\right\} .
\end{aligned}
$$

The foregoing quantities will be used throughout the remainder of Section 5 . 


\subsection{Perishable capacity units}

In accordance with the general framework, we will say that early planning, meaning planning on the first available capacity unit, is preferred in stage $N-k$ if

$$
y_{N-k}(1+i)+E\left[f_{N-k-1}((0,0,1),(0,0,1))\right] \geq y_{N-k}+E\left[f_{N-k-1}((0,1,0),(0,0,1))\right] .
$$

We obtain the following result:

Theorem 4. Within the general framework, in the absence of reinvestment revenues $(i=0)$ and in case of infinite time lags $\left(l_{z}=\infty, z=A, B\right)$, early planning is preferred if the cost of a non-regular capacity unit is larger than $y_{B}$, else the planner remains indifferent to the chosen order plan.

Logically, early available capacity units cannot give rise to more allocation opportunities than later ones and thus have a larger risk of perishing without being used.

Previously, we have shown in Theorem 3 and Corollary 1 that order plans that allocate perishing capacity units are favored. Likewise, Theorem 4 suggests that in the absence of reinvestment revenues and in case of infinite time lags, we need only consider order plans that plan early in time.

\subsection{Finite maximal time lags}

When we restrict the length of the maximal time lags, Theorem 4 no longer applies. The reason is that capacity units from early time periods do not necessarily generate fewer allocation opportunities than units from later periods. As an example we present rush orders, which are order types with higher payoffs in combination with smaller time lags than other order types.

A rush order with a maximal time lag of one can only be planned at its release time, so that, for a specific capacity unit, the probability of receiving this rush order depends on the number of available capacity units in the same period and not on the time until the unit perishes. Under these circumstances, planning other order types on the early capacity unit may no longer be the best option. This intuition is confirmed by our analysis based on the general framework from Section 5.1. Order type $A$ is considered to be a rush order, so that $y_{A}>y_{B} \wedge l_{A}<l_{B}$. For convenience, we set $l_{A}=1, l_{B}>3$ and $i=0$ to eliminate 
the influence of reinvestment revenues. From the general framework, we learn that early planning does not prevail since

$$
E\left[f_{N-2}((0,0,1),(0,0,1))\right]<E\left[f_{N-2}((0,1,0),(0,0,1))\right]
$$

for cases (a) and (b): the resulting profile on the right side of the equation has more opportunities to accept high-payoff rush orders. In case (c), both options are equally good as a consequence of equal opportunities for accepting project $A$ in both resulting capacity profiles. These equations remain valid for offers in stage $N-4$.

Building on the foregoing paragraph, we advance that companies that are confronted with rush orders benefit from a levelled capacity profile, which is a profile with more or less equal allocation opportunities in every period. This can be illustrated through a comparison of the profits resulting from a non-levelled profile $\mathbf{x}_{N-2}^{N L}=((0,0,2)(0,0,0))$ and levelled $\mathbf{x}_{N-2}^{L}=((0,1,1)(0,0,0))$. In the presence of a rush order type $A$ with $l_{A}=1$ (and $\left.i=0\right)$, it holds that $E\left[f_{N-2}\left(\mathbf{x}_{N-2}^{L}\right)\right]>E\left[f_{N-2}\left(\mathbf{x}_{N-2}^{N L}\right]\right.$, which implies that the levelled profile leads to better results, whereas the company would remain neutral in relation to both profiles if the time lags of both types were sufficiently large.

\subsection{Reinvestment revenues}

When the interest rate $i$ has a strictly positive value, reinvestment revenues can be reaped. In stage $N-3$ of the general framework, early planning is best in all three cases from the general framework when

$$
y_{N-3}>\operatorname{Pr}[A] \cdot y_{A}+\operatorname{Pr}[B] \cdot \bar{y}
$$

In addition, early planning is preferred if $c \geq y_{B}$ for very small interest rates. For incoming projects in stage $N-4$, arrival $\mathbf{w}_{N-4}$ is planned early if

$$
y_{N-4}>\operatorname{Pr}[A] \cdot y_{A}+\operatorname{Pr}[B] \cdot\left(\operatorname{Pr}[A] \cdot y_{A}+\operatorname{Pr}[B] \cdot \bar{y}\right)
$$

Early planning is also best when $c \geq y_{B}$ and the interest rate is near zero. The righthand side of the two foregoing equations constitutes a cut-off value on the payoff of the incoming project, above which early planning is preferred; one could speak of high-versus low-payoff projects. We conclude that, when the interest rate $i$ has a positive value, high payoffs generate high reinvestment revenues and are thus better planned early in time, while low-payoff projects are pushed later in time to leave room for other projects. 
We also observe that the cut-off value for early planning increases with the number of stages $N$ in the planning horizon. This observation is related to the fact that the value function of our SDP (as given by Eq. (3)) is non-decreasing with $N$.

\subsection{Non-regular capacity units}

The presented models support the quantification of the value of having non-regular capacity units at one's disposal. This can be useful when the price of keeping non-regular capacity available needs to be negotiated with subcontractors.

As an example we again consider the rush-order case from Section 5.3. When calculating the difference between the expected value of case (b) and (a), we derive the value of the non-regular capacity unit in stage $N-3$. If $y_{B}>c$ then $E\left[f_{N-2}((0,1,1),(0,0,1))\right]-$ $E\left[f_{N-2}((0,1,1),(0,0,0))\right]=\operatorname{Pr}[B] \cdot\left(y_{B}-c\right)$ and if $y_{B} \leq c$ then $E\left[f_{N-2}((0,1,1),(0,0,1))\right]-$ $E\left[f_{N-2}((0,1,1),(0,0,0))\right]=0$, so that the availability of a non-regular capacity unit is valuable only if the unit hiring cost is smaller than the payoff of project $B$. In this setting, the value of the non-regular capacity unit is independent of $y_{A}$, which may seem counterintuitive. The reason is simply that the rush order under examination can only be executed at its release time so that only orders of type $B$ can be associated with the non-regular capacity unit in question.

\subsection{Valuation of flexible due dates}

Our models also allow for the valuation of increased flexibility under the form of an extension of the maximal time lag. For an illustration we examine the example of Section 4.3. Suppose that the deadline of the project $\mathbf{w}_{1}$ under consideration were increased by one time period. This would result in two additional eligible order plans, namely $\mathbf{a}_{1}^{3}=((0,0,1),(1,0,0))$ and $\mathbf{a}_{1}^{4}=((0,1,1),(0,0,0))$. As a consequence, $f_{1}((0,2,1),(1,0,0))$ would change into $\max \{\{20.82 ; 25.32 ; 21.8\} \cup\{28.88 ; 26.32\}\}$. The value of the increase in flexibility is 3.56 , which represents the increase in the expectation at time 0 of the value at time $T$ of the selected portfolio. When using this value during negotiations with the customer (e.g. for granting discounts), the time value of money should obviously be taken into account. 


\section{Computational performance of the model}

The presented SDP approach has the advantage of easily dealing with many different problem characteristics (varying due dates, hiring non-regular capacity units, precedence relations,...). A downside to the high flexibility is the large problem size, reflected in the numerous variables in the state vector. This causes the dynamic program to blow up and become unmanageable for all but relatively small problem sizes. Fortunately, as was demonstrated in the first illustration (see Section 4.2), it is not always necessary to calculate all possible states in order to solve the model.

The number of states of the solution method can be obtained by summing the number of states per stage over all stages. From Section 3.3, we know that the number of possible states in each stage is non-increasing with the stage number. An upper bound for the number of states in stage $k$ is the number of different capacity profiles, calculated as:

$$
\prod_{j=r_{k}+1}^{T} m_{j}+\sum_{j=1}^{T-r_{k}}\left(\begin{array}{c}
T-r_{k} \\
j
\end{array}\right) \widehat{m}_{k}^{\left(T-r_{k}-j\right)}\left(\widehat{s}_{k}+1\right)^{j}
$$

with $\widehat{m}_{k}$ and $\widehat{s}_{k}$ representing the maximal number of the per-period regular and non-regular capacity units between time $r_{k}$ and $T$, respectively. The first part of Eq. (5) represents the number of capacity profiles for which the regular capacity is not exhausted $\left(m_{t}>0\right)$ in any period, while the second part is an upper bound for the number of profiles for which no regular capacity units are available during at least one period. In periods with depleted regular capacity units, non-regular capacity can be allocated until exhaustion. The index $j$ represents the number of periods for which the regular capacity is depleted. The binomial coefficient counts the number of ways of picking $j$ unordered outcomes (depleted periods) from $T-r_{k}$ possibilities (periods). An upper bound on the total number of states is obtained by summing Eq. (5) from $k=2$ to $k=N$. Since there is only one state to consider in stage 1 , we increase the result by 1 .

\section{Conclusions and further research}

In this paper, we have investigated dynamic order acceptance and planning in an overdemanded multi-project organization that aims at maximizing its profits. We have stressed the importance of integrating order acceptance and capacity planning in order to be able to live up to competitive due dates and reduce the sometimes excessive use of highly expensive 
non-regular capacity. We have used stochastic dynamic programming to maximize the expected profits of the company within the planning horizon. Our exact methods have allowed us to gain valuable insights into how the problem characteristics influence the acceptance and capacity-planning decisions. We have separated and quantified the influence of a number of problem characteristics, such as perishable capacity units, finite maximal time lags, reinvestment revenues and non-regular capacity units.

We have established that without reinvestment revenues and with infinite deadlines, one need only consider early planning. When the maximal time lags become restrictive, this policy is no longer optimal; when companies are confronted with rush orders, for instance, it makes more sense to strive for a levelled capacity profile. In general, the best planning policy aims at reducing the risk of having to reject short-lagged projects because of a lack of available capacity units. The effect of reinvestment revenues on the planning decision has also been investigated. We conclude that high payoffs generate high reinvestment revenues and are thus better planned early; our model allows us to determine the cut-off between high- and low-payoff projects. In the final paragraphs, we have quantified the gains from non-regular capacity units and from due-date flexibility.

Since the size of the dynamic program has the tendency to blow up quickly, further research is needed if optimal planning solutions are to be developed for realistically-sized problems. We are convinced that the models and insights described in this paper can serve as guidelines in this process. In these future models, it would be interesting to incorporate the possibility of replanning once a project is accepted. Within this extension, we could view the estimated realization time of the project as a due date which can be deviated from at a contract-specified lateness cost.

\section{Appendix: proofs}

Proof (Theorem 1): We assume that $\mathbf{x}_{k}$ is a state vector with at least one unit of regular and non-regular capacity available in time period $q$. Order plan $\mathbf{a}_{k}^{j}$ allocates a unit of regular capacity from period $q . \mathbf{a}_{k}^{\tilde{j}}$ is associated with a similar order plan, for which the unit of regular capacity from period $q$ is replaced with a unit of non-regular capacity from the same period. Following Definition 1, two cases need to be considered.

Case 1: $k=N$. From Eq. (2), the reward function equals $y_{k}(1+i)^{\left(T-t_{j}^{*}\right)}-c s_{j}^{*}$, since we know that $j \neq 0$. The first term remains the same for both order plans. The second term, 
however, is smaller for order plan $a_{N}^{\tilde{j}}$ because of the additional cost $c$ of the non-regular capacity unit.

Case 2: $k \neq N$. As was established for Case 1, the reward for both order plans differs with a value of $c$. The theorem is thus proven if:

$$
E\left[f_{k+1}\left(\mathbf{x}_{k+1}\right)\right]+c \geq E\left[f_{k+1}\left(\tilde{\mathbf{x}}_{k+1}\right)\right]
$$

We assume that $\mathbf{x}_{k+1}$ and $\tilde{\mathbf{x}}_{k+1}$ are the state vectors after implementing order plan $\mathbf{a}_{k}^{j}$ and $\mathbf{a}_{k}^{\tilde{j}}$, respectively. In every later stage $k+l$, we choose the best order plan $\tilde{\mathbf{a}}_{k+l}^{h}$ and the (except for at most one capacity unit) similar order plan $\mathbf{a}_{k+l}^{h}$ from the respective decision sets $\tilde{\mathcal{A}}_{k+l}$ and $\mathcal{A}_{k+l}$. This strategy only maximizes the value of $f_{k+l}\left(\tilde{\mathbf{x}}_{k+l}\right)$ while it derives a lower bound for $f_{k+l}\left(\mathbf{x}_{k+l}\right)$. Below, we list all arguments to the max-operator of Eq. (3) for the two decision sets.

\begin{tabular}{|c|c|c|c|}
\hline \multicolumn{2}{|r|}{$\tilde{\mathcal{A}}_{k+l}$} & \multicolumn{2}{|r|}{$\mathcal{A}_{k+l}$} \\
\hline $\begin{array}{l}\tilde{\mathbf{a}}_{k+l}^{0}: \\
\tilde{\mathbf{a}}_{k+l}^{1}:\end{array}$ & $\begin{array}{c}E\left[f_{k+l+1}\left(\tilde{\mathbf{x}}_{k+l+1}\right)\right] \\
g_{k+l}\left(\tilde{\mathbf{a}}_{k+l}^{1}, \mathbf{w}_{k+l}\right)+ \\
E\left[f_{k+l+1}\left(v\left(\mathbf{x}_{k+l}-\mathbf{a}_{k+l}^{1}\right)\right)\right]\end{array}$ & $\begin{array}{l}\mathbf{a}_{k+l}^{0}: \\
\mathbf{a}_{k+l}^{1}:\end{array}$ & $\begin{array}{c}E\left[f_{k+l+1}\left(\mathbf{x}_{k+l+1}\right)\right] \\
g_{k+l}\left(\tilde{\mathbf{a}}_{k+l}^{1}, \mathbf{w}_{k+l}\right)-c+ \\
E\left[f_{k+l+1}\left(v\left(\mathbf{x}_{k+l}-\mathbf{a}_{k+l}^{1}\right)\right)\right]\end{array}$ \\
\hline$\tilde{\mathbf{a}}^{n}$ & & & $\left(\tilde{\mathbf{a}}^{n} \quad \cdots\right.$ \\
\hline$\tilde{\mathbf{a}}_{k+l}^{n}$ & $\begin{array}{c}g_{k+l}\left(\tilde{\mathbf{a}}_{k+l}^{n}, \mathbf{w}_{k+l}\right)+ \\
E\left[f_{k+l+1}\left(v\left(\mathbf{x}_{k+l}-\mathbf{a}_{k+l}^{n}\right)\right)\right]\end{array}$ & $\mathbf{a}_{k+l}^{n}:$ & $\begin{array}{c}g_{k+l}\left(\tilde{\mathbf{a}}_{k+l}^{n}, \mathbf{w}_{k+l}\right)-c+ \\
E\left[f_{k+l+1}\left(v\left(\mathbf{x}_{k+l}-\mathbf{a}_{k+l}^{n}\right)\right)\right]\end{array}$ \\
\hline$\tilde{\mathbf{a}}_{k+l}^{n+1}:$ & $\begin{array}{c}g_{k+l}\left(\mathbf{a}_{k+l}^{n+1}, \mathbf{w}_{k+l}\right)+ \\
E\left[f_{k+l+1}\left(v\left(\tilde{\mathbf{x}}_{k+l}-\tilde{\mathbf{a}}_{k+l}^{n+1}\right)\right)\right]\end{array}$ & $\mathbf{a}_{k+l}^{n+1}:$ & $\begin{array}{c}g_{k+l}\left(\mathbf{a}_{k+l}^{n+1}, \mathbf{w}_{k+l}\right)+ \\
E\left[f_{k+l+1}\left(v\left(\mathbf{x}_{k+l}-\mathbf{a}_{k+l}^{n+1}\right)\right)\right]\end{array}$ \\
\hline$\tilde{\mathbf{a}}_{k+l}^{M}:$ & $\begin{array}{c}g_{k+l}\left(\mathbf{a}_{k+l}^{M}, \mathbf{w}_{k+l}\right)+ \\
E\left[f_{k+l+1}\left(v\left(\tilde{\mathbf{x}}_{k+l}-\tilde{\mathbf{a}}_{k+l}^{M}\right)\right)\right]\end{array}$ & $\mathbf{a}_{k+l}^{M}:$ & $\begin{array}{c}g_{k+l}\left(\mathbf{a}_{k+l}^{M}, \mathbf{w}_{k+l}\right)+ \\
E\left[f_{k+l+1}\left(v\left(\mathbf{x}_{k+l}-\mathbf{a}_{k+l}^{M}\right)\right)\right]\end{array}$ \\
\hline
\end{tabular}

Three types of order plans exist: degenerate plans $(h=0)$, plans that allocate a regular capacity unit from period $q$ in $\tilde{\mathbf{a}}_{k+l}^{h}$ and a non-regular unit from the same period in $\mathbf{a}_{k+l}^{h}$ $(h=1,2, \ldots, n)$ and remaining order plans $(h=n+1, \ldots, M)$. If $\mathbf{a}_{k+l}^{h}$, with $h=1,2, \ldots, n$; is chosen and results in a negative reward $g_{k+l}\left(\tilde{\mathbf{a}}_{k+l}^{h}, \mathbf{w}_{k+l}\right)-c$, the reward is set to 0 . Theorem 2 confirms that we still derive a lower bound.

In order to prove Eq. A1) we examine all possible situations.

Step 0: set $l=1$.

Step 1: if the capacity units from period $q$ have perished while entering stage $k+l$ then the state vectors $\tilde{\mathbf{x}}_{k+l}$ and $\mathbf{x}_{k+l}$ are equal, so that Eq. (A1) is true. If stage $k+l=N$ then we combine Eq. (2) and Eq. A1): $E\left[g_{N}\left(\mathbf{a}_{N}^{h}, \mathbf{w}_{N}\right)\right]+c \geq E\left[g_{N}\left(\tilde{\mathbf{a}}_{N}^{h}, \mathbf{w}_{N}\right)\right]$. Since the non-regular 
unit can only be assigned in case $\mathbf{y}_{N}>c$, the first term will always be larger than or equal to the second term.

Step 2: The chosen order plan belongs to one of the three groups;

Case 1: $h=0$; Set $l:=l+1$. Go to step 1 .

Case $2: h \in\{1,2, \ldots, n\}$; the order plans only differ in their stage $k+l$ reward. The logic of step 1 proves Eq. (A1).

Case 3: $h \in\{n+1, n+2, \ldots, M\}$; the immediate rewards from these order plans are equal. Set $l:=l+1$. Go to step 1 .

Proof (Lemma 1): The lemma states that $E\left[f_{k}\left(\mathbf{x}_{k}\right)\right] \geq E\left[f_{k}\left(\tilde{\mathbf{x}}_{k}\right)\right]$ if $\mathbf{x}_{k}>_{l} \tilde{\mathbf{x}}_{k}$.

Case 1: $k=N$; the lemma holds if $\max _{\mathbf{a}_{N}^{j} \in \mathcal{A}_{N}}\left\{g_{N}\left(\mathbf{a}_{N}^{j}, \mathbf{w}_{N}\right)\right\} \geq \max _{\tilde{\mathbf{a}}_{N}^{\tilde{j}} \in \tilde{\mathcal{A}}_{N}}\left\{g_{N}\left(\tilde{\mathbf{a}}_{N}^{\tilde{j}}, \mathbf{w}_{N}\right)\right\}$, according to Eq. (3). Since $\mathbf{x}_{k}>_{l} \tilde{\mathbf{x}}_{k}$, the set of eligible order plans $\tilde{\mathcal{A}}_{N} \subseteq \mathcal{A}_{N}$, such that $g_{N}\left(\mathbf{a}_{N}^{j}, \mathbf{w}_{N}\right) \geq g_{N}\left(\tilde{\mathbf{a}}_{N}^{\tilde{j}}, \mathbf{w}_{N}\right)$.

Case 2: $k \neq N$; we follow the same reasoning as for Case 1. From Eq. (3), we derive that $E\left[f_{k}\left(\mathbf{x}_{k}\right)\right] \geq E\left[f_{k}\left(\tilde{\mathbf{x}}_{k}\right)\right]$ if $\max _{\mathbf{a}_{k}^{j} \in \mathcal{A}_{k}}\left\{g_{k}\left(\mathbf{a}_{k}^{j}, \mathbf{w}_{k}\right)+E\left[f_{k+1}\left(\mathbf{x}_{k+1}\right)\right]\right\} \geq \max _{\tilde{\mathbf{a}}_{k}^{j} \in \tilde{\mathcal{A}}_{k}}\left\{g_{k}\left(\tilde{\mathbf{a}}_{k}^{\tilde{j}}, \mathbf{w}_{k}\right)+\right.$ $\left.E\left[f_{k+1}\left(\tilde{\mathbf{x}}_{k+1}\right)\right]\right\}$. Since $\mathbf{x}_{k}$ is the larger state, it follows that $\tilde{\mathcal{A}}_{k} \subseteq \mathcal{A}_{k}$. The maximum over all eligible order plans in the decision set $\tilde{\mathcal{A}}_{k}$ can therefore never be larger than the maximum over all order plans in decision set $\mathcal{A}_{k}$.

Proof (Theorem 2): Suppose order plan $\mathbf{a}_{k}^{q}(q \neq 0)$ results in an immediate non-positive reward: $g_{k}\left(\mathbf{a}_{k}^{q}, \mathbf{w}_{k}\right) \leq 0$. Following Definition 1 two cases need to be considered.

Case 1: $k=N$; since rejection has a zero reward and $g_{N}\left(\mathbf{a}_{N}^{q}, \mathbf{w}_{N}\right) \leq 0$, the first case of Definition 1 is established.

Case 2: $k \neq N$; since $g_{k}\left(\mathbf{a}_{k}^{0}, \mathbf{w}_{k}\right)=0$ accordance with Definition 1 is shown if:

$$
g_{k}\left(\mathbf{a}_{k}^{q}, \mathbf{w}_{k}\right)+E\left[f_{k+1}\left(v\left(\mathbf{x}_{k}-\mathbf{a}_{k}^{q}\right)\right)\right] \leq E\left[f_{k+1}\left(v\left(\mathbf{x}_{k}\right)\right)\right]
$$

Since $q \neq 0, \mathbf{a}_{k}^{q}$ must allocate a positive number of capacity units. Therefore, $\mathbf{x}_{k}>_{l} \mathbf{x}_{k}-\mathbf{a}_{k}^{q}$. Based on the definition of the perishing function $v$ in Eq. (4), we conclude that $\mathbf{x}_{k+1} \geq_{l} v\left(\mathbf{x}_{k}-\right.$ $\left.\mathbf{a}_{k}^{q}\right)$. From Lemma 11 we know that $E\left[f_{k+1}\left(\mathbf{x}_{k+1}\right)\right] \geq E\left[f_{k+1}\left(v\left(\mathbf{x}_{k}-\mathbf{a}_{k}^{q}\right)\right)\right]$ if $\mathbf{x}_{k+1}>_{l} v\left(\mathbf{x}_{k}-\mathbf{a}_{k}^{q}\right)$. In case $\mathbf{x}_{k+1}=v\left(\mathbf{x}_{k}-\mathbf{a}_{k}^{q}\right)$, we can easily establish that $E\left[f_{k+1}\left(\mathbf{x}_{k+1}\right)\right]=E\left[f_{k+1} v\left(\left(\mathbf{x}_{k}-\mathbf{a}_{k}^{q}\right)\right)\right]$ following the reasoning of the same theorem. Since $g_{k}\left(\mathbf{a}_{k}^{q}, \mathbf{w}_{k}\right) \leq 0$, Eq. A2 is valid.

Proof (Theorem 3): The reward function from Eq. (2) is $y_{k}(1+i)^{\left(T-t_{j}^{*}\right)}-c s_{j}^{*}$. The first term of the reward function can be affected by changing to an order plan with a different 
finish time $t_{j}^{*}$. Since perishing capacity units must be, by definition, situated in the first periods of the time line, $t_{j}^{*}$ can never be decreased by replacing capacity units with other non-perishing capacity units. Therefore the first term of the reward function cannot increase by switching to $\mathbf{a}_{k}^{\tilde{j}}$. The second term of the reward function equals the cost of non-regular resource usage. Since no non-regular capacity units from order plan $\mathbf{a}_{k}^{j}$ may be replaced with regular capacity units in order plan $\mathbf{a}_{k}^{\tilde{j}}$, the second term cannot decrease through a switch of plans. From this discussion we conclude that

$$
g_{k}\left(\mathbf{a}_{k}^{j}, \mathbf{w}_{k}\right) \geq g_{k}\left(\mathbf{a}_{k}^{\tilde{j}}, \mathbf{w}_{k}\right), \text { for } k=1, \ldots, N .
$$

Case 1: $k=N$; following Definition 1, the validity of the theorem is established in Eq. (A3). Case 2: $k \neq N$; the theorem is valid if

$$
g_{k}\left(\mathbf{a}_{k}^{j}, \mathbf{w}_{k}\right)+E\left[f_{k+1}\left(v\left(\mathbf{x}_{k}-\mathbf{a}_{k}^{j}\right)\right)\right] \geq g_{k}\left(\mathbf{a}_{k}^{\tilde{j}}, \mathbf{w}_{k}\right)+E\left[f_{k+1}\left(v\left(\mathbf{x}_{k}-\mathbf{a}_{k}^{\tilde{j}}\right)\right)\right] .
$$

Since order plan $\mathbf{a}_{k}^{j}$ has more units that perish in stage $k+1$ and both order plans must allocate the same number of units, $v\left(\mathbf{x}_{k}-\mathbf{a}_{k}^{j}\right)$ is larger than $v\left(\mathbf{x}_{k}-\mathbf{a}_{k}^{\tilde{j}}\right)$. Eq. A4 follows from Lemma 1 and Eq. (A3).

Proof (Corollary 1): In case $\mathcal{A}_{k}=\mathcal{F}_{k} \cup\left\{\mathbf{a}_{k}^{0}\right\}$, any eligible order plan that allocates nonperishing capacity units while leaving regular perishing capacity units unallocated, can be replaced by another eligible order plan for which a number of the allocated non-perishing capacity units are replaced by perishing regular capacity units. Theorem 3 states that the second order plan dominates the first one.

Proof (Theorem 4): The theorem is proven by induction on the number of stages. As initial step, we wish to show that

$$
E\left[f_{N-2}((0,0,1),(0,0,1))\right] \geq E\left[f_{N-2}((0,1,0),(0,0,1))\right] .
$$

Using the general framework, it is easy to establish that:

$$
\begin{aligned}
& \text { If } c \leq y_{B} \quad \Rightarrow E\left[f_{N-2}((0,0,1),(0,0,1))\right]=E\left[f_{N-2}((0,1,0),(0,0,1))\right], \\
& \text { otherwise } \Rightarrow E\left[f_{N-2}((0,0,1),(0,0,1))\right]>E\left[f_{N-2}((0,1,0),(0,0,1))\right] .
\end{aligned}
$$

The second part of the proof consists in demonstrating the validity of the induction step: $E\left[f_{N-k}((0, \ldots, 0,1),(0, \ldots, 0,1))\right] \geq E\left[f_{N-k}((0, \ldots, 1,0),(0, \ldots, 0,1))\right] \Rightarrow$ 
$E\left[f_{N-k-1}((0, \ldots, 0,1),(0, \ldots, 0,1))\right] \geq E\left[f_{N-k-1}((0, \ldots, 1,0),(0, \ldots, 0,1))\right]$.

The induction step can be written as:

$$
\begin{aligned}
& \sum_{z=A, B} \operatorname{Pr}[z] \cdot \max \left\{\begin{array}{ll}
E\left[f_{N-k+1}((0, \ldots, 0,1),(0, \ldots, 0,1))\right] & {\left[\mathbf{a}_{N-k}^{0}\right]} \\
y_{z}+E\left[f_{N-k+1}((0, \ldots, 0,0),(0, \ldots, 0,1))\right] & {\left[\mathbf{a}_{N-k}^{1}\right]}
\end{array}\right\} \geq \\
& \sum_{z=A, B} \operatorname{Pr}[z] \cdot \max \left\{\begin{array}{ll}
E\left[f_{N-k+1}((0, \ldots, 1,0),(0, \ldots, 0,1))\right] & {\left[\tilde{\mathbf{a}}_{N-k}^{0}\right]} \\
y_{z}+E\left[f_{N-k+1}((0, \ldots, 0,0),(0, \ldots, 0,1))\right] & {\left[\tilde{\mathbf{a}}_{N-k}^{1}\right]} \\
y_{z}-c+E\left[f_{N-k+1}((0, \ldots, 1,0),(0, \ldots, 0,0))\right] & {\left[\tilde{\mathbf{a}}_{N-k}^{2}\right]}
\end{array}\right\} \\
& \Rightarrow \\
& \begin{array}{c}
\sum_{z=A, B} \operatorname{Pr}[z] \cdot \max \left\{\begin{array}{ll}
E\left[f_{N-k}((0, \ldots, 0,1),(0, \ldots, 0,1))\right] & {\left[\mathbf{a}_{N-k-1}^{0}\right]} \\
y_{z}+E\left[f_{N-k}((0, \ldots, 0,0),(0, \ldots, 0,1))\right] & {\left[\mathbf{a}_{N-k-1}^{1}\right]}
\end{array}\right\} \geq \\
\sum_{z=A, B} \operatorname{Pr}[z] \cdot \max \left\{\begin{array}{ll}
E\left[f_{N-k}((0, \ldots, 1,0),(0, \ldots, 0,1))\right] & {\left[\tilde{\mathbf{a}}_{N-k-1}^{0}\right]} \\
y_{z}+E\left[f_{N-k}((0, \ldots, 0,0),(0, \ldots, 0,1))\right] & {\left[\tilde{\mathbf{a}}_{N-k-1}^{1}\right]} \\
y_{z}-c+E\left[f_{N-k}((0, \ldots, 1,0),(0, \ldots, 0,0))\right] & {\left[\tilde{\mathbf{a}}_{N-k-1}^{2}\right]}
\end{array}\right\} .
\end{array}
\end{aligned}
$$

Case 1: $c \geq y_{A}$; due to Theorem 2 the allocation of the non-regular capacity unit $\left(\tilde{\mathbf{a}}_{k}^{2}\right)$ need not be considered. The validity of the induction step is based on two arguments. First of all, planning on the regular capacity unit $\left(\mathbf{a}_{k}^{1}\right.$ and $\left.\tilde{\mathbf{a}}_{N-k}^{1}\right)$ results in the same reward $y_{z}+E\left[f_{N-k}((0, \ldots, 0,0),(0, \ldots, 0,1))\right]$ for both capacity profiles. Secondly, the induction hypothesis states that in case of rejection, $E\left[f_{N-k}((0, \ldots, 0,1),(0, \ldots, 0,1))\right]$ is larger than or equal to $E\left[f_{N-k}((0, \ldots, 1,0),(0, \ldots, 0,1))\right]$.

Case 2: $c<y_{A}$; using the argumentation from case 1, the theorem is proven if we demonstrate that $y_{z}+E\left[f_{N-k}((0, \ldots, 0,0),(0, \ldots, 0,1))\right] \geq y_{z}-c+E\left[f_{N-k}((0, \ldots, 1,0),(0, \ldots, 0,0))\right]$. We again consider two cases: $c<y_{B}$ and $c \geq y_{B}$.

If $c<y_{B}$, it follows that:

$$
\begin{aligned}
& E\left[f_{N-k}((0, \ldots, 0,0),(0, \ldots, 0,1))\right] \geq-c+E\left[f_{N-k}((0, \ldots, 1,0),(0, \ldots, 0,0))\right] \\
& \quad \Rightarrow\left(y_{A}-c\right) \cdot \operatorname{Pr}[A] \cdot\left(1-(\operatorname{Pr}[B])^{k+1}\right) / \operatorname{Pr}[A]+\left(y_{B}-c\right) \cdot(\operatorname{Pr}[B])^{k+1} \geq \\
& \quad-c+y_{A} \cdot \operatorname{Pr}[A] \cdot\left(1-(\operatorname{Pr}[B])^{k}\right) / \operatorname{Pr}[A]+y_{B}(\operatorname{Pr}[B])^{k} \\
& \quad \Rightarrow 0 \geq\left(y_{B}-y_{A}\right) \cdot(\operatorname{Pr}[B])^{k} \cdot \operatorname{Pr}[A] .
\end{aligned}
$$

If $c \geq y_{B}$, we do not accept project $B$ because of Theorem 2 , so that

$$
\begin{aligned}
& E\left[f_{N-k}((0, \ldots, 0,0),(0, \ldots, 0,1))\right] \geq-c+E\left[f_{N-k}((0, \ldots, 1,0),(0, \ldots, 0,0))\right] \\
& \quad \Rightarrow\left(y_{A}-c\right) \cdot \operatorname{Pr}[A] \cdot\left(1-(\operatorname{Pr}[B])^{k+1}\right) / \operatorname{Pr}[A] \geq-c+y_{A} \cdot \operatorname{Pr}[A] \cdot\left(1-(\operatorname{Pr}[B])^{k}\right) / \operatorname{Pr}[A] \\
& \quad \Rightarrow \operatorname{Pr}[B] \cdot c \geq y_{B}-y_{A} \cdot \operatorname{Pr}[A] .
\end{aligned}
$$




\section{References}

Adler, P., Mandelbaum, A., Nguyen, V. \& Schwerer, E. (1995). From project to process management: an empirically-based framework for analyzing product development time. Management Science, 41(3), pp 458-484.

Akkan, C. (1997). Finite-capacity scheduling-based planning for revenue-based capacity management. European Journal of Operational Research, 100, pp 170-179.

Baker, N. \& Freeland, J. (1975). Recent advances in R\&D benefit measurement and project selection methods. Management Science, 21(10), pp 1164-1175.

Balakrishnan, N., Patterson, J. \& Sridharan, S. (1996). Rationing capacity between two product classes. Decision Sciences, 27(2), pp 185-214.

Bard, J., Balachandra, R. \& Kaufman, P. (1988). An interactive approach to R\&D project selection and termination. IEEE Transactions on Engineering Management, $35(3)$, pp 139-146.

Beaujon, G., Martin, S. \& McDonald, G. (2001). Balancing and optimizing a portfolio of R\&D projects. Naval Research Logistics, 48(1), pp 18-40.

Bertsekas, D. (2005). Dynamic programming and optimal control. Vol. 1. Athena Scientific.

Brenner, M. (1994). Practical R\&D project prioritization. Research Technology Management, $37(5)$, pp 38-42.

Cooper, R., Edgett, S. \& Kleinschmidt, E. (1999). New product portfolio management: Practices and performance. Journal of Product Innovation Management, 16(4), pp 333351.

Czajkowski, A. \& Jones, S. (1986). Selecting interrelated R\&D projects in space planning technology. IEEE Transactions on Engineering Management, 33(1), pp 17-24.

De Boer, R. (1998). Resource-constrained Multi-project Management - A hierarchical decision support system. PhD thesis. University of Twente, Enschede, the Netherlands.

De, P., Ghosh, J. \& Wells, C. (1993). Job selection and sequencing on a single machine in a random environment. European Journal of Operational Research, 70, pp 425-431. 
De Reyck, B. (1998). Scheduling projects with generalized precedence relations. Exact and heuristic procedures. PhD thesis. Department of applied economics, Katholieke Universiteit Leuven.

Demeulemeester, E. \& Herroelen, W. (2002). Project scheduling - A research handbook. Kluwer Academic Publishers, Dordrecht.

Dickinson, M., Thornton, A. \& Graves, S. (2001). Technology portfolio management: optimizing interdependent projects over multiple time periods. IEEE Transactions on Engineering Management, 48(4), pp 518-527.

Ebben, M., Hans, E. \& Olde Weghuis, F. (2005). Workload based order acceptance in job shop environments. OR Spektrum, 27, pp 107-122.

Eilat, H., Golany, B. \& Shtub, A. (2006). Constructing and evaluating balanced portfolios of R\&D projects with interactions: a DEA based methodology. European Journal of Operational Research, 172, pp 1018-1039.

Fox, G., Baker, N. \& Bryant, L. (1984). Economic models for R\&D project selection in the presence of project interactions. Management Science, 30, pp 890-902.

Golabi, K. (1987). Selecting a group of dissimilar projects for funding. IEEE Transactions on Engineering Management, 34(3), pp 138-145.

Hall, D. \& Nauda, A. (1990). An interactive approach for selecting R\&D projects. IEEE Transactions on Engineering Management, 37, pp 126-133.

Hans, E. (2001). Resource Loading by Branch-and-Price Techniques. PhD thesis. University of Twente, Enschede, the Netherlands.

Henriksen, A. \& Traynor, A. (1999). A practical R\&D project-selection scoring tool. IEEE Transactions on Engineering Management, 46(2), pp 158-170.

Jørgensen, T. (1999). Project scheduling as a stochastic dynamic decision problem. PhD thesis. Norwegian university of Science and Technology, Trondheim, Norway.

Kavadias, S. \& Loch, C. (2004). Project Selection under Uncertainty. Dynamically Allocating Resources to Maximize Value. Kluwer. 
Kerzner, H. (1997). Project management: a systems approach to planning, scheduling, and controlling. John Wiley \& Sons.

Kis, T. (2005). A branch-and-cut algorithm for scheduling of projects with variable-intensity activities. Mathematical Programming, 103(3), pp 515-539.

Kleywegt, A. \& Papastavrou, J. (1998). The dynamic and stochastic knapsack problem. Operations Research, 46(1), pp 17-35.

Kleywegt, A. \& Papastavrou, J. (2001). The dynamic and stochastic knapsack problem with random sized items. Operations Research, 49(1), pp 26-41.

Levy, N. \& Globerson, S. (1997). Improving multiproject management by using a queuing theory approach. Project Management Journal, 28(4), pp 40-46.

Lewis, H. \& Slotnick, S. (2002). Multi-period job selection: planning work loads to maximize profit. Computers $\& 5$ Operations Research, 29, pp 1081-1098.

Lewis, M., Ayhan, H. \& Foley, R. (1999). Bias optimality in a queue with admission control. Probability in the Engineering and Informational Sciences, 13, pp 309-327.

Loch, C., Pich, M., Terwiesch, C. \& Urbschat, M. (2001). Selecting R\&D projects at BMW: A case study of adopting mathematical programming models. IEEE Transactions on Engineering Management, 48(1), pp 70-80.

Luenberger, D. (1998). Investment Science. Oxford University Press.

Perry, T. \& Hartman, J. (2004). Allocating manufacturing capacity by solving a dynamic, stochastic multiknapsack problem. Research Report ISE 04T-009. Lehigh University PA.

Ringuest, J., Graves, S. \& Case, R. (2004). Mean-gini analysis in R\&D portfolio selection. European Journal of Operational Research, 154, pp 157-169.

Ross, K. \& Tsang, D. (1989). The stochastic knapsack problem. IEEE Transactions on Communications, 37(7), pp 740-747.

Ross, S. (1983). Introduction to stochastic dynamic programming. Academic Press, Inc. 
Saaty, T. (1994). How to make a decision: the analytic hierarchy process. Interfaces, 24(6), pp 19-43.

Slotnick, S. \& Morton, T. (1996). Selecting jobs for a heavily loaded shop with lateness penalties. Computers \& Operations Research, 23, pp 131-140.

Weatherford, L. \& Bodily, S. (1992). A taxonomy and research overview of perishableasset revenue management: yield management, overbooking and pricing. Operations Research, 40(5), pp 831-844.

Weber, R., Werners, B. \& Zimmerman, H. (1990). Planning models for research and development. European Journal of Operations Research, 48, pp 175-188.

Wester, F., Wijngaard, J. \& Zijm, W. (1992). Order acceptance strategies in a production-toorder environment with setup times and due-dates. International Journal of Production Research, 30(6), pp 1313-1326.

Wheelwright, S. \& Clark, K. (1992). Creating project plans to focus product development. Harvard Business Review, pp 70-82.

Yang, K.-K. \& Sum, C.-C. (1997). An evaluation of due date, resource allocation, project release, and activity scheduling rules in a multi-project environment. European Journal of Operational Research, 103, pp 139-154.

Zijm, W. (2000). Towards intelligent manufacturing planning and control systems. OR Spektrum, 22, pp 313-345. 